\title{
A novel nano zero-valent iron@chitosan for effective removal of Microcystin-LR: kinetics and adsorption mechanism
}

\author{
Xiaofan Yang ${ }^{1}$, Xueyu Wei ${ }^{2 *}$, Xiaoping $\mathrm{Xu}^{2}$, Zhigang Liu ${ }^{3}$ \\ ${ }^{1}$ College of biological and chemical engineering, Anhui Polytechnic University, 241000 Wuhu, P.R. China \\ ${ }^{2}$ The school of civil engineering and architecture, Anhui Polytechnic University, 241000 Wuhu, P.R. China \\ ${ }^{3}$ Ningbo tap water Co., Ltd, 315041 Ningb, P.R. China
}

\begin{abstract}
Microcystin-LR (MC-LR) is one of the most notorious toxins liberated from cyanobacteria in drinking water sources. In this study, a skillful method access to new nanozero-valent iron@ @chitosan (nZVIMC) was synthesized by a facile one step method. The as-prepared nZVIMC was employed as an adsorbent for the effective removal MC-LR from aqueous solution. Transmission electron microscopy (TEM) demonstrates that nZVIMC is in quasi-spherical shape with size of around $50 \mathrm{~nm}$, effect of variable parameters such as $\mathrm{pH}$, contact time, initial concentration of MC-LR and adsorption properties of nZVIMC on MC-LR was further investigated. Scanning electron microscope (SEM) reveals that the particles are nearly spherical in shape with agglomeration. The results indicated that good adsorption performance was achieved at an initial $\mathrm{pH}$ of 5 . The adsorption kinetics of nZVIMC was better fitted by pseudo-secondorder kinetics. The adsorption isotherm data was fitted well to Langmuir isotherm and then to Freundlich model, with an adsorption capacity of $68.9 \mathrm{mg} / \mathrm{g}$ at $300 \mathrm{~K}$. Thus, we believe that nZVIMC can be used as a low cost material for efficient removal of MC-LR from water.
\end{abstract}

\section{Introduction}

Algal bloom caused by eutrophication has grown up to be a serious environmental problem, such as the Taihu lake pollution incident encountered in Wuxi city of China during $2007^{[1]}$. Microcystin-LR (MC-LR) is a part of the deadliest algae toxins produced by cyanobacteria [2]. The World Health Organization has set a safe guideline value of $1.0 \mu \mathrm{g} / \mathrm{L}$ of MC-LR in daily drinking water ${ }^{[3,4]}$. The toxicity of MC-LR is explained by their strong binding and inhibition of protein phosphatases. MC-LR is also known to promote the growth of tumor and has a genotypic effect as well as strong mutagenicity. Long term consumption of MC-LR contaminated water may induce liver damage, liver cancer, and even death in humans ${ }^{[5,6]}$.

Although inhibiting Microcystis (MCs) growth in water resources is the most effective method to reduce MCs, it needs long-term investment. Therefore, expeditious removal of MCs is another option. Although variety of treatment methods, including activated carbon absorption, potassium permanganate oxidation, $\mathrm{TiO}_{2}$ photocatalytic oxidation, and sand filter biodegradation have been reported for the removal of MCs [add reference], some appreciable disadvantages have been identified. Removal of MC-LR by activated carbon is a simple and effective technology, but the removal efficiency is significantly reduced in natural water sources due to the presence of other organics [7]. Treatment with potassium permanganate could destroy
MCs quickly; however, it easily interferes with unsaturated compounds, which lead to rapid depletion of potassium permanganate and incomplete oxidation of $\mathrm{MCs}^{[8]}$. Though most of the MCs in waters are eliminated through biodegradation ${ }^{[9-10]}$, the process takes long time to implement this technology in practical water treatment process.

The aim of this research was tantamount to design a new material for removal of MCs with high efficiency and to reduce risk to human health. The prepared material is rapid, cost-effective end reproducible.

\section{Materials}

MC-LR and $\mathrm{FeCl}_{3} \cdot 6 \mathrm{H}_{2} \mathrm{O}$ (Ferric tetrachloride) were purchased from Sigma-Aldrich; chitosan with a deacetylation degree of $80-95 \%$ was purchased from Sino pharm Chemical Reagent Co.Ltd., China. All other chemicals were of analytical grade and deionized water was used for all experiments. Required concentrations of the standard solution were obtained by diluting stock solution with double distilled water.

\subsection{Preparation of nZVIMC}

The synthesis of nZVIMC was induced by chitosan hydrogel via in-situ co-precipitation method at low temperature and normal atmosphere. Chitosan hydrogel was prepared by dissolving $0.5 \mathrm{~g}$ of chitosan in $200 \mathrm{~mL}$

\footnotetext{
Corresponding author:wxyu1027@126.com
} 
of $0.5 \%(\mathrm{v} / \mathrm{v})$ acetic acid solution with continuous stirring. $5.2 \mathrm{~g}$ of $\mathrm{FeCl}_{3} \cdot 6 \mathrm{H}_{2} \mathrm{O}$, were dissolved in $50 \mathrm{~mL}$ of distilled water, and honey was added dropwise as a reducing agent to convert $\mathrm{Fe}^{3+}$ to $\mathrm{Fe}^{0}$, then the mixture was stirred vigorously for $30 \mathrm{~min}$. Chitosan hydrogel were added to $\mathrm{Fe}^{0}$ solution by stirring at $3000 \mathrm{rpm}$ for 10 min in a water bath at $40^{\circ} \mathrm{C}$ to form chitosan-iron ions composites. It was provided sufficient time and proper conditions for chitosan to chelate iron ions. After stirring, the obtained product was washed three times with ethanol and filtered. The obtained product was dried in a vacuum oven at $90^{\circ} \mathrm{C}$ for overnight and the final product was stored in a stoppered bottle.

\subsection{Adsorbent Characterization}

Morphology and grain size of nZVIMC were examined by a Tecnai G20 TEM (FEI, USA) and S4800 SEM (Hitachi, Japan). Magnetic behavior of the nanoparticles sample was obtained by a MPMS-XL-7 magnetic measurement instrument (quantum design, USA).

\subsection{Adsorption experiments}

All the adsorption tests were conducted in $250 \mathrm{~mL}$ conical flasks. For adsorption kinetics, $1 \mathrm{mg}$ of nZVIMC was added to $100 \mathrm{~mL}$ of MC-LR $(300 \mu \mathrm{g} / \mathrm{L})$ and shaken at $150 \mathrm{rpm}\left(25^{\circ} \mathrm{C}\right)$ for $120 \mathrm{~min}$. while for adsorption isotherms test, $1 \mathrm{mg}$ of nZVIMC was added to 50$1000 \mu \mathrm{g} / \mathrm{L}$ of MC-LR in a conical flask and was shaken for $120 \mathrm{~min}$ at $150 \mathrm{rpm}$ and at $25^{\circ} \mathrm{C}$ of temperature. The adsorption equilibrium time was based on the results of adsorption kinetics. The concentration of MC-LR was identified by high performance liquid chromatography tandem mass spectrometry ${ }^{[11]}$.

The effect of $\mathrm{pH}$ on MC-LR adsorption was investigated by adjusting the initial solution $\mathrm{pH}$ from 2 to 12 using $0.1 \mathrm{~mol} / \mathrm{L} \mathrm{HCl}$ and $0.1 \mathrm{~mol} / \mathrm{L} \mathrm{NaOH}$ with initial MC-LR concentration of $300 \mu \mathrm{g} / \mathrm{L}$. Zeta potentials were measured with a Zetasizer Nano ZS90 instrument (Malven Instruments Ltd., UK).

\section{Results and Discussion}

\subsection{Characterization of $n Z$ VIMC}

As shown in Fig.1, the isoelectric point of nZVIMC was $\mathrm{pH}$ 7.8. the morphology of synthesized nZVIMC was investigated by TEM and SEM imaging as shown in Fig.2. From the figure it indicates that nZVIMC particles are in quasi-spherical in shape with size of around $50 \mathrm{~nm}$ (Fig. 2a). From SEM images (Fig. 2b) it indicates that there is a slight aggregation of these nanoparticles due to the existence of cross-linked chitosan on the surface of nZVIMC.

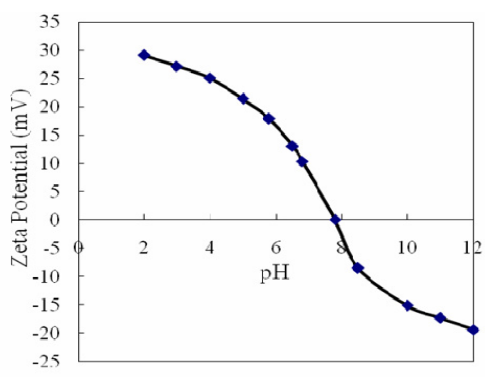

Fig.1. Zeta potential of nZVIMC $(1 \mathrm{~g} / \mathrm{L})$ as a function of $\mathrm{pH}$.

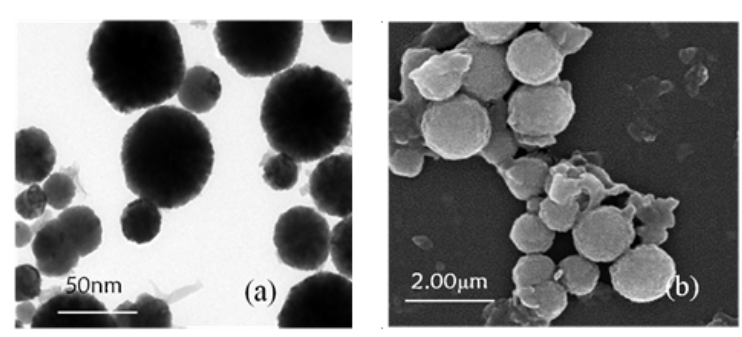

Fig. 2. TEM (a) and SEM (b) images of nZVIMC.

Magnetic behavior of the nanoparticles sample was obtained using a MPMS-XL-7 magnetic measurement instrument (Quantum Design, USA). The super paramagnetic character of nZVIMC is evident from the nanoparticles magnetic hysteresis loop as shown in Fig. 3. The nanoparticles saturation magnetization was 59.53 $\mathrm{emu} / \mathrm{g}$. This demonstrated that the adsorbent can be separated from its aqueous dispersion easily in a few minutes using an external applied magnetic field.

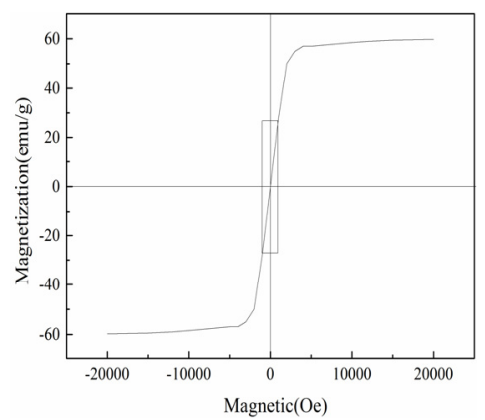

Fig.3. Magnetic hysteresis loop of nZVIMC.

Adsorption time is the key parameter because it can reflect the adsorption kinetics of an adsorbent. Fig.2a shows the contact time profile of MC-LR removal of nZVIMC. The adsorption amount of MC-LR increased sharply within the first $1 \mathrm{~h}$ of contact time, after which was slowed down. This phenomenon may be the adsorption sites were void and adsorbates could be easily interacted with these sites during the initial stage. With increase in time the remaining vacant sites are not easily occupied, until the system reached equilibrium.

To determine the adsorption kinetics of MC-LR on the nanoparticles, two kinetic models were applied to fit the experimental data. Pseudo-first-order and pseudosecond-order which were used to explain the solid/liquid adsorption.

In order to investigate the controlling mechanism of the adsorption process, the adsorption/time data obtained 
were applied to three kinetic models, including the pseudo-first-order model, pseudo-second-order model, and intraparticle diffusion model ${ }^{[12,13]}$. The Lagergren equation was used as the pseudo-first-order kinetic model by the following equation:

$$
\log \left(\mathrm{q}_{\mathrm{e}}-q_{t}\right)=\log q_{e}-\frac{k_{1}}{2.303} t
$$

where $q_{e}$ and $q_{t}(\mathrm{mg} / \mathrm{g})$ are the amount of MC-LR adsorbed on ACMCSM at equilibrium and at a given time $t$, respectively; $k_{l}$ is the pseudo-first-order rate constant $\left(\mathrm{min}^{-1}\right)$.

The pseudo-second-order kinetic model was as follows:

$$
\frac{t}{q_{t}}=\frac{1}{k_{2} q_{e}^{2}}+\frac{t}{\mathrm{q}_{\mathrm{e}}}
$$

where $k_{2}(\mathrm{~g} /(\mathrm{mg} \min ))$ is the adsorption rate constant of the pseudo-second-order.

The intrapaticle diffusion model was given by the follows:

$$
q_{t}=k_{p} t^{1 / 2}+C
$$

where $q_{e}\left(\mathrm{mg} / \mathrm{g} \min ^{0.5}\right)$ is the intrapaticle diffusion rate constant and $C$ of adsorption constant is the intercept.

Table1. Best-Fit Model for MC-LR Adsorption on nZVIMC

\begin{tabular}{|c|c|c|c|}
\hline & Param.1 & Param.2 & $\mathrm{R}^{2}$ \\
\hline First-order & $q_{e}(\mathrm{mg} / \mathrm{g})=28.75$ & $k_{l}=0.005$ & 0.941 \\
\hline Second-order & $q_{e}(\mathrm{mg} / \mathrm{g})=48.38$ & $k_{2}=0.017$ & 0.997 \\
\hline Intrapaticle & $/$ & $K_{p}=3.049$ & 0.893 \\
\hline Langmuir & $q_{m}(\mathrm{mg} / \mathrm{g})=72.99$ & $b=0.0995$ & 0.997 \\
\hline Freundlich & $\mathrm{K}_{\mathrm{F}}=12.9591$ & $n=2.592$ & 0.944 \\
\hline
\end{tabular}

The results of the kinetics were obtained by analyzing Fig.4. Based on the correlation coefficients, the pseudo-second-order kinetic equation with $\mathrm{R}^{2}>0.99$ was feasible to describe the adsorption process, as shown in Table 1. This suggests that the overall process seems to be controlled by chemisorption without any mass transfer in the solution ${ }^{[12]}$. These results are in good agreement with the adsorbing pharmaceuticals in drinking water ${ }^{[14]}$.

\subsection{Adsorption isotherm}

In order to interpret the adsorption data, Langmuir and Freundlich adsorption isotherm models were used to describe the equilibrium adsorption for MC-LR on nZVIMC.

Linearized Langmuir equation is ${ }^{[15]}$ :

$$
\frac{c_{e}}{q_{e}}=\frac{1}{b q_{m}}+\frac{c_{e}}{\mathrm{q}_{\mathrm{m}}}
$$

Where $q_{e}$ and $c_{e}$ are the amount adsorbed $(\mathrm{mg} / \mathrm{g})$ and the adsorbate concentration in solution $(\mathrm{mg} / \mathrm{L})$, both at equilibrium $b(\mathrm{~L} / \mathrm{mg})$ is a constant related to the heat of adsorption and $q_{m}(\mathrm{mg} / \mathrm{g})$ is the maximum adsorption capacity for monolayer formation on adsorbent.
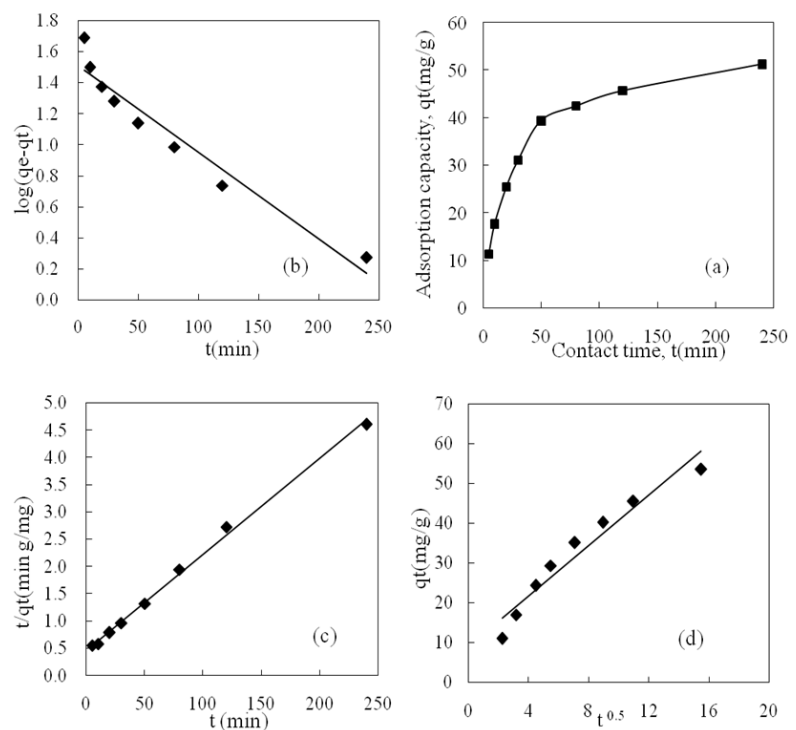

Fig.4. Effect of contact time on MC-LR adsorption (a); fitting pseudo-first-order kinetic plots (b \& c) and intra-particle diffusion kinetic plots (d) of MC-LR adsorption, in which $t$ is the reaction time $(\mathrm{min})$ and $\mathrm{qt}(\mathrm{mg} / \mathrm{g})$ is the amount of adsorbed fluoride at a particular reaction time $t$.

The Freundlich isotherm is an empirical equation employed to describe heterogeneous systems in which the model is expressed by the following equation ${ }^{[16]}$ :

$$
\ln q_{e}=\ln K_{F}+\frac{1}{\mathrm{n}} \ln C_{e}
$$

Where $K_{F}$ is Freundlich constant and $n$ is the heterogeneity factor.

The theoretical parameters of adsorption isotherms along with regression coefficients are summarized in Table 1. From the two studied systems (Fig 5), the Langmuir isotherm model correlated better $\left(\mathrm{R}^{2}>0.99\right)$ than Freundlich isotherm with the experimental data from adsorption equilibrium of MC-LR by MACFM, The maximum adsorption values were in good accordance with experimentally obtained values. The fact that the Langmuir isotherm model assumes monolayer adsorption on a surface with a finite number of identical sites, these sites are energetically equivalent and there is no interaction between adsorbed molecules. It has been reported that the values of $n$ in the range 1-10 indicates favorable condition for adsorption ${ }^{[17]}$. In the present work, the exponent was around 2.592, predicting the adsorption system is well.

The removal efficiency of toxins was calculated based on the adsorption isotherm kinetics. Approximately $90 \%$ of toxins were removed at the concentrations ranging from 50 to $500 \mu \mathrm{g} / \mathrm{L}$. which is same as the reported toxin levels in drinking water ${ }^{[11]}$. The removal efficiency was comparable with other chemical or biological methods ${ }^{[18,19]}$, satisfied the maximum allowable concentration (1000ng/L) prescribed by the world health organization (WHO) in human drinking water ${ }^{[5]}$. 

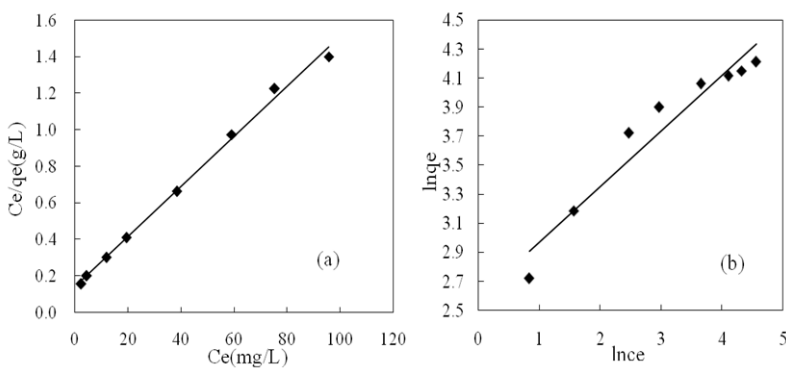

Fig.5. Linear plots of Langmuir (a) and Freundlich adsorption isotherm (b).

\subsection{Effect of solution pH on MC-LR adsorption}

As an important parameter in water chemistry, $\mathrm{pH}$ can significantly affect MC-LR adsorption. We could know from Fig.6 that as the $\mathrm{pH}$ increased, positive charge of MACFM decreased and negative charged increased. While above $\mathrm{pH}$ 2.1 MC-LR was negative charged ${ }^{[20]}$, MC-LR was easily removed, because nZVIMC and MCLR own the opposite charge to attract each other except at $\mathrm{pH} 2$. In addition, under acidic conditions, the hydrophobicity of MC-LR and nZVIMC was much stronger than in alkaline conditions. The similar hydrophobicities of these molecules under acidic conditions may also contribute to the high removal efficiency.

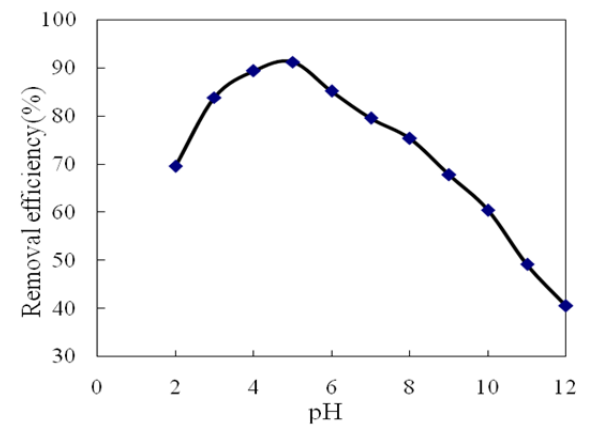

Fig.6. Effect of $\mathrm{pH}$ on MC-LR removal efficiency (initial MC$\mathrm{LR}$ concentration $300 \mu \mathrm{g} / \mathrm{L}$; temperature $25^{\circ} \mathrm{C}$; contact time $60 \mathrm{~min}$ ) at the different $\mathrm{pH}$.

\section{Conclusion}

We have synthesized a core-shell type nZVIMC, with a magnetic core and carboxymethy cellulase shell decorated with zero iron for effective removal of MCLR from water as an eco-friendly and cost-effective water purification technique. The magnetite carboxymethy cellulase microspheres displayed a spherical shape with $\mathrm{Fe} 0$ decorated on the surface of the microsphere. The mechanism for the adsorption involved electrostatic interaction, hydrogen bonding and complex action reaction. The optimum $\mathrm{pH}$ range for MC-LR removal was from 3 to 9 and the interference by natural organic matter was low, demonstrating that this adsorbent can be used in a natural water environment.

\section{Acknowledgments}

This work was supported by the Natural Science Fund of Education Department of Anhui province (TSKJ2016B016), the Advanced Research for the National Natural Science Foundation of China (zryy1310) and Teaching Quality Research Project of Anhui Polytechnic University(2017jyxm31).

\section{References}

1. X.J.Zhang, C.Chen, P.F.Lin, A.X.Hou, Z.B.Niu, J.Wang, Environ. Sci. Technol. 45,1(2011)

2. R. Al-Ammar, A. Nabok, A. Hashim, T. Smith, Sens Actuators B Chem. 209,31(2015)

3. WHO, Guidelines for drinking-water quality (2011)

4. B. Žegura A. Štraser, M. Filipič, Mutation Res. 727, 1-2(2011)

5. J.A. Westrick, D.C. Szlag, Anal. Bioanal. Chem. 397, 5 (2010)

6. L. Chen, D.D. Dionysiou, K. O’Shea, Environ. Sci. Technol. 45, 6 (2011)

7. W.J.Huang, , B.L. Cheng, Y.L.Cheng, Journal of Hazardous Materials. 141, 1 (2007)

8. J.Pietsch, K.Bornmann, W. Schmidt, Acta Hydroch. Hydrob. 30,1 (2002)

9. D.G. Bourne, R.L. Blakeley, P. Riddles, Water Res. 40, 6 (2006)

10. G. Grutzmacher, G. Wessel, S.Klitzke, I.Chorus, Environ. Sci. Technol. 44, 2 (2010), 657-662.

11. S. M. Azevedo, W.W. Carmichael, E. M. Jochimsen, K.L. Rinehart, S. Lau, G.R. Shaw, G.K. Eaglesham, Toxicology 181-182, (2002)

12. Y.S.Ho, G. McKay, Water Res. 34, 3(2000)

13. M. Yurdakoc, Y. Scki, S. K. Yuedakoc, J. Colloid Interface Sci. 286, 2(2005)

14. X. Hu, L. Mu, Q. Zhou, J. Wen, J. Pawliszyn, Environ. Sci. Technol. 45, 11(2011)

15. I. Langumuir, J. Am. Chem. Soc. 40,(1918)

16. N. Ünlü, M. Ersoz, J. Hazard. Mater. 136, 2 (2006)

17. Y. Bulut, N. Gozubenli, H. Aydin, J. Hazard. Mater. 144, 1-2(2007)

18. L. Ho, A.L. Gaudieux, S. Fanok, G. Newcombe, A.R. Humpage, Toxicon, 50, 3(2007)

19. J. Li, K. Shimizu, M.K. Sakharkar, M. Utsumi, Z. Zhang, N. Sugiura, Bioresour. Technol. 102, 20(2011)

20. L.A. Lawton, P.K.J. Robertson, B. Cornish, I.L. Marr, M. Jaspars, Journal of Catalysis 213, 1 (2003) 